http://dx.doi.org/10.32911/as.2018.v11.n2.580

Aporte Santiaguino 11(2), julio-diciembre 2018: 263-274

ISSN-L 2616-9541

\title{
Evaluación de la vulnerabilidad sísmica de las edificaciones de la zona urbana del distrito de Chiquián, utilizando el model builder del ArcGIS
}

\author{
Evaluation of the seismic vulnerability of the buildings in the urban area of \\ the district Chiquian, using the ArcGIS model builder
}

Tito Tinoco Meyhuay ${ }^{1}$, Javier Cotos Vera ${ }^{1}$ y Remo Bayona Antúnez ${ }^{1}$

\section{RESUMEN}

El presente trabajo de investigación descriptivo, no experimental y transversal, tiene como objetivo evaluar la vulnerabilidad sísmica de las edificaciones de la zona urbana del distrito de Chiquián, aplicando el model builder del ArcGIS.

La metodología propuesta permite la automatización y la creación de un modelo de vulnerabilidad sísmica utilizando la herramienta model builder del ArcGIS, donde se evaluó once parámetros para la determinación del índice de vulnerabilidad y el índice de daños en las edificaciones de la zona urbana del distrito de Chiquián.

De un total de 1417 viviendas se encontraron que el 14,7\% de las viviendas están en vulnerabilidad baja, el 21,2\% en vulnerabilidad media, el 48,8\% en vulnerabilidad alta y el 15,2\% en vulnerabilidad muy alta. Además, teniendo en cuenta el índice de vulnerabilidad y una aceleración horizontal del terreno de $0,32 \mathrm{~g}$, se calcularon que 1209 viviendas $(85,32 \%)$ sufrían el colapso total y 208 viviendas (14,68\%) seguirían en pie con algún daño estructural. Según el índice de daño calculado y teniendo en cuenta el precio por metro cuadrado de construcción, si ocurriera un sismo con las características descritas anteriormente los daños económicos se cuantificarían a la suma de $\mathrm{S}$. $70,043,266.14$

Palabras clave: sismo; vulnerabilidad sísmica; modelamiento; índice de vulnerabilidad; índice de daños.

\footnotetext{
1 Universidad Nacional Santiago Antúnez de Mayolo. Huaraz, Perú.

(C) Los autores. Este artículo es publicado por la Revista Aporte Santiaguino de la Universidad Nacional Santiago Antúnez de Mayolo. Este es un artículo de acceso abierto, distribuido bajo los términos de la Licencia Creative Commons Atribución-NoComercial-CompartirIgual 4.0 Internacional. (http://creativecommons.org/licenses/ by-nc-sa/4.0/), que permite el uso no comercial, distribución y reproducción en cualquier medio, siempre que la obra original sea debidamente citada.
} 


\section{ABSTRACT}

The present descriptive, non-experimental and transversal investigation work has the objective of evaluate the seismic vulnerability of the buildings of the urban area of the district of Chiquian applying the AcrGIS model builder.

The proposed methodology allows the automation and the creation of a model of seismic vulnerability using the ArcGIS model builder, where eleven parameters were evaluated for the determination of the vulnerability index and the damage index in the buildings of the urban area of the district of Chiquian.

Of an overall of 1417 buildings, it was found that the $14,7 \%$ of the houses are in low vulnerability, the $21 \%$ in medium vulnerability, the $48,8 \%$ in high vulnerability and the $15,2 \%$ in very high vulnerability. Besides, considering the vulnerability index and a horizontal acceleration of the ground of $0,32 \mathrm{~g}$, it was calculated that 1209 houses $(85,32 \%)$ suffered the total collapse and 208 houses (14,68\%) will keep standing with some structural damage. According to the index of calculated damage and considering the price per square meter of building, if an earthquake would occur, with the previously described characteristics

Keywords: earthquake; seismic vulnerability; modeling; vulnerability index; damage index.

\section{INTRODUCCIÓN}

Saputra et al. (2017), realizaron un estudio al sureste de la ciudad de Yogyakarta, debido a la ocurrencia de un terremoto de 6,3 grados que produjo daños y pérdidas por más de S/ 3,100,000. Los resultados muestran que los edificios más vulnerables son las estructuras de mampostería reforzada con techos de teja de arcilla y los edificios más seguros son las de estructuras de mamposteria reforzada con techos de asbesto o techos de zinc.

Senouci et al. (2018), realizaron una investigación referida a la representación gráfica de los escenarios de daños y pérdidas por terremotos, a través de las estimaciones del índice de vulnerabilidad y las herramientas SIG que permitan una mejor planificación urbana. Los resultados muestran que las diferentes zonas de Orán (Argelia) tienen cierta homogeneidad en su respuesta sísmica y las zonas más vulnerables que requieren de una recalificación urbana.

De la Cruz (2011) realizó un estudio de peligro sísmico probabilístico y espectro uniforme en la región Ancash, siendo la investigación de tipo histórico y descriptivo que compara resultados obtenidos con otros trabajos anteriores. Concluye que la región Ancash es una zona de alta sismicidad y alto riesgo, debido a las construcciones muy precarias en la mayor parte de las provincias. 
Marín (2012), realizó una evaluación del riesgo sísmico del centro histórico de la ciudad de Huánuco, aplicó el método del índice de vulnerabilidad de Benedetti y Petrini y el sistema de información geográfica. El estudio comprendió el análisis de 3266 edificaciones que conforman la zona de estudio, para cada edificación se estimó el estado de la edificación, características estructurales y su tiempo de antigüedad. Los resultados muestran que gran parte de las edificaciones evaluadas presentan una vulnerabilidad baja a media. Finalmente, se determinó las pérdidas directas en función del índice de daños y el costo de la edificación.

La zona de estudio se encuentra ubicada en el distrito de Chiquián, Provincia de Bolognesi- Región Ancash y según los antecedentes históricos corresponde a una zona de alta sismicidad (Ministerio de Vivienda, Construcción y Saneamiento, 2016), Norma E-030, Mapa de Zonificación Sísmica (Zona 3) y que en el año de 1970 fue totalmente destruida, con más de 75,000 muertes a lo largo del callejón de Huaylas; por lo que es latente el riesgo de ocurrencia de un evento sísmico igual o superior. Por otro lado, el crecimiento desordenado y sin planificación en la zona urbana del distrito de Chiquián, sin criterios antisísmicos, falta de dirección y control en las construcciones nuevas, mantenimiento deficiente en las construcciones existentes, presencia de viviendas de adobe con más de 30 años de antigüedad, incrementan la vulnerabilidad física; observándose que, la mayoría de las viviendas y la infraestructura de servicio social se construyen con nula o baja calidad de diseño y materiales locales, sin una adecuada asesoría técnica y limitándose en la mayoría de casos a contratar mano de obra no calificada.

En el presente trabajo se utilizó la metodología del índice de vulnerabilidad sísmica citado por (Chavarría y Gómez, 2001), cuyo modelo geoespacial fue creado con la herramienta model builder del ArcGIS teniendo en cuenta las consideraciones de (Enviromental Systems Research Institute, 2016), que permitió el análisis de 1417 viviendas de la zona urbana del distrito de Chiquián. Se generaron once mapas temáticos que corresponden a cada parámetro evaluado y mediante la aplicación del modelo geoespacial de vulnerabilidad sísmica se obtuvo el mapa final del índice de vulnerabilidad sísmica de la zona urbana Chiquián.

El presente trabajo de investigación contribuirá a la prevención, en función del índice de vulnerabilidad sísmica, el índice de daños y las pérdidas económicas, por lo que se convertirá en u instrumento de gestión del gobierno local del distrito de Chiquián.

\section{MATERIAL Y MÉTODOS}

La población estuvo representada por 1417 edificaciones de la zona urbana del distrito de Chiquián, siendo la muestra igual al total de las edificaciones. Las coordenadas de referencia de la Plaza de Armas en el datum WGS84 son: latitud sur: 1009'7.31", longitud oeste: $77^{\circ} 09^{\prime} 24.66^{\prime \prime}$ y una altitud de 3,407 msnm. 
La información se obtuvo utilizando libretas de campo, fichas de vulnerabilidad, imágenes satelitales y el plano catastral levantado por COFOPRI, los cuales fueron georreferenciados y homogenizados en el datum WGS84, zona 18S. Así mismo, se hicieron calicatas para la obtención de muestras de suelos.

El proceso metodológico consistió en la generación de la cartografía de la zona urbana del Distrito de Chiquián, muestreo de suelos e interpretación, generación del modelo de vulnerabilidad sísmica con el model builder del ArcGIS, aplicación del formulario de vulnerabilidad, cálculo del índice de vulnerabilidad, elaboración de mapas temáticos y el cálculo del índice de daños y pérdidas económicas.

En la figura 1 se observa el flujograma que permitió la creación del modelo de vulnerabilidad sísmica, en función de las características estructurales y no estructurales de las edificaciones de la zona urbana del distrito de Chiquián.

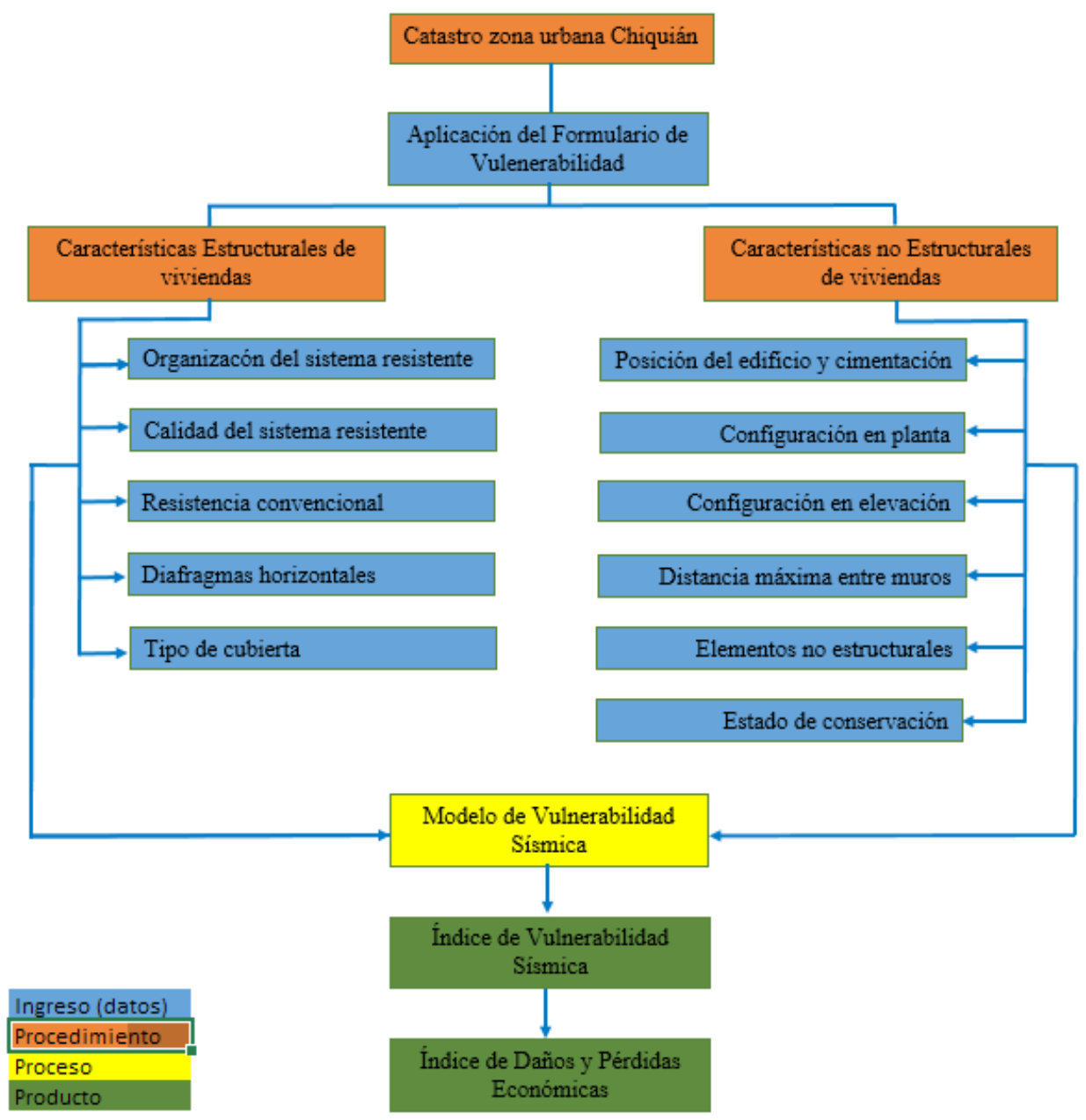

Figura 1. Flujograma para la obtención del modelo de índice de vulnerabilidad 


\section{RESULTADOS}

\section{Información básica}

El Distrito de Chiquián tiene una extensión de 184,165 Km². Según el INEI, Censos Nacionales 2007 XI de Población y VI de Vivienda, la población del Distrito de Chiquián es de 4,087 habitantes. Su clima es característico de la sierra del Perú, con una temperatura anual de $10^{\circ}$ a $25^{\circ} \mathrm{C}$ y una precipitación anual de $650 \mathrm{~mm}$ a $950 \mathrm{~mm}$. Según el estudio de suelos se ha encontrado diferentes tipos de suelos como grava arcillosa, arena limo arcillosa con grava, grava arcillosa con arena, arena arcillosa, grava mal graduada limosa, etc. cuyas capacidades portantes fluctúan entre 0,40 y 1,40 Kg/ $\mathrm{cm}^{2}$, clasificándose de baja a media resistencia.

\section{Elaboración de la cartografía}

La cartografía de la zona urbana del distrito de Chiquián fue elaborado teniendo en cuenta el Plano Catastral levantado por COFOPRI en el año 2012, el levantamiento de datos con GPS e imágenes satelitales de Google Earth en coordenadas UTM, Zona 18 Sur del esferoide internacional, los cuales fueron homogenizados al datum WGS 84.

\section{Estudio de suelos}

Para determinar las características geo mecánicas del suelo de fundación de la zona urbana del distrito de Chiquián se realizaron 7 calicatas realizando la interpolación correspondiente y la generación del mapa de capacidad portante de los suelos.

\section{Creación del modelo de vulnerabilidad sísmica}

El modelo de vulnerabilidad sísmica fue desarrollado en el entorno ArcGIS y utilizando la herramienta model builder, permitiendo la automatización de los procesos y la obtención del índice de vulnerabilidad sísmica en la zona urbana del distrito de Chiquián, tal como se observa en la figura 2. 


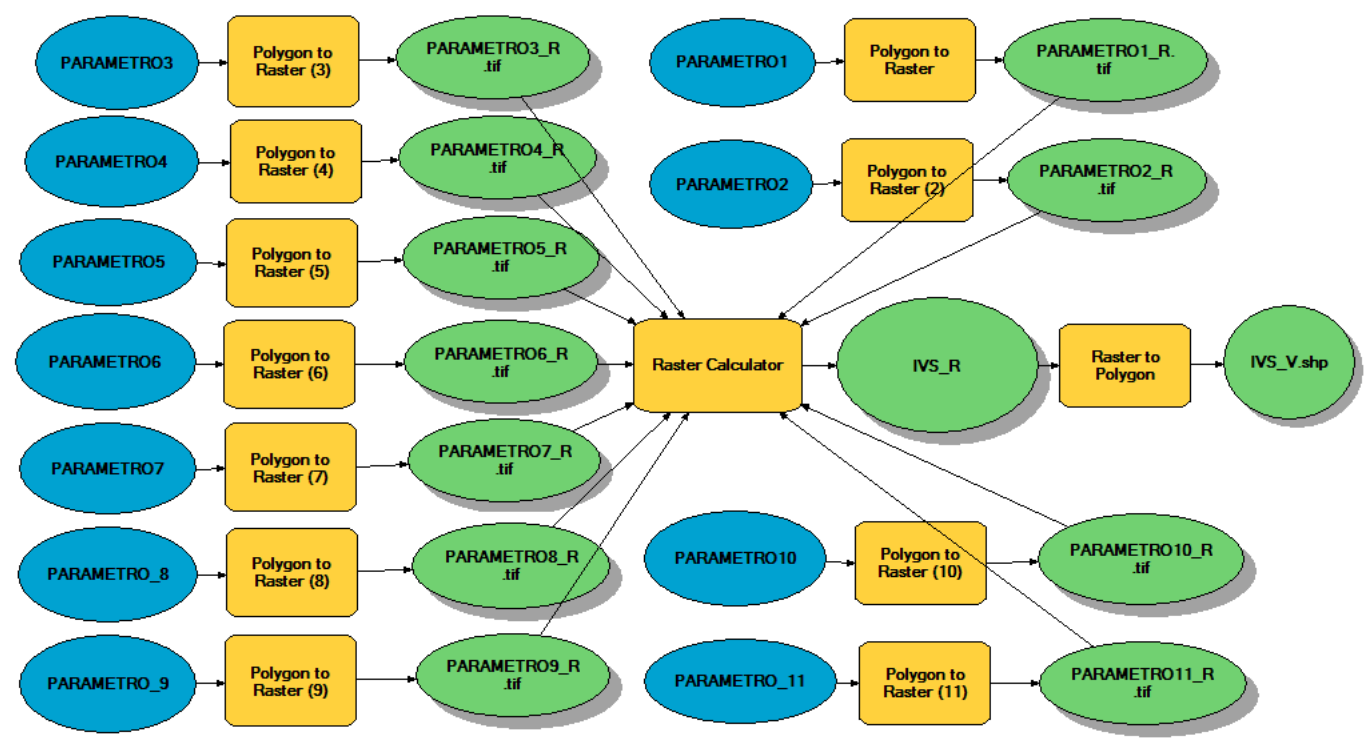

Figura 2. Modelo de vulnerabilidad sísmica

Teniendo en cuenta la cartografía de la zona urbana del distrito de Chiquián y las fichas de encuesta (formulario de vulnerabilidad), adaptado de (Chavarría y Gómez, 2001) se analizaron 1417 edificaciones, obteniéndose el resultado que se muestra en la tabla 1.

Tabla 1: Número de edificaciones por parámetros y clases

\begin{tabular}{clrrrrr}
\hline \multirow{2}{*}{$\begin{array}{c}\text { No } \\
\text { Parámetro }\end{array}$} & \multicolumn{3}{c}{ Descripción } & N & \multicolumn{3}{c}{ edificaciones por clase } \\
& & B & C & \multicolumn{2}{c}{ D } \\
\hline 1 & Organización del sistema resistente & 195 & 14 & 1002 & 206 \\
2 & Calidad del sistema resistente & 125 & 87 & 988 & 217 \\
3 & Resistencia convencional & 196 & 15 & 933 & 273 \\
4 & Posición del edificio y cimentación & 45 & 1149 & 220 & 3 \\
5 & Diafragma horizontal & 160 & 50 & 993 & 214 \\
6 & Configuración en planta & 172 & 43 & 993 & 209 \\
7 & Configuración en elevación & 169 & 43 & 994 & 211 \\
8 & Distancia máxima entre los muros & 186 & 27 & 993 & 211 \\
9 & Tipo de cubierta & 167 & 47 & 989 & 214 \\
10 & Elementos no estructurales & 193 & 21 & 991 & 212 \\
11 & Estado de conservación & 442 & 38 & 649 & 288 \\
\hline
\end{tabular}




\section{Cálculo del índice de vulnerabilidad}

Para el cálculo del índice de vulnerabilidad se elaboraron once mapas temáticos que corresponden a cada uno de los parámetros estudiados y aplicando el modelo de vulnerabilidad sísmica se obtuvo el Mapa de Índice de Vulnerabilidad (figura 3).

Los índices de vulnerabilidad obtenidos fueron agrupados en los siguientes rangos de clasificación, tal como se observa en la tabla 2.

Tabla 2: Clasificación de edificaciones según el índice de vulnerabilidad

\begin{tabular}{ccccc}
\hline Clase & Rango Iv & Vulnerabilidad & No edificaciones & \% \\
\hline A & $<90$ & Baja & 208 & 14.7 \\
B & $91-180$ & Media & 301 & 21.2 \\
C & $181-270$ & Alta & 692 & 48.8 \\
D & $>271$ & Muy alta & 216 & 15.3 \\
& Total & & $\mathbf{1 4 1 7}$ & $\mathbf{1 0 0 . 0}$ \\
\hline
\end{tabular}

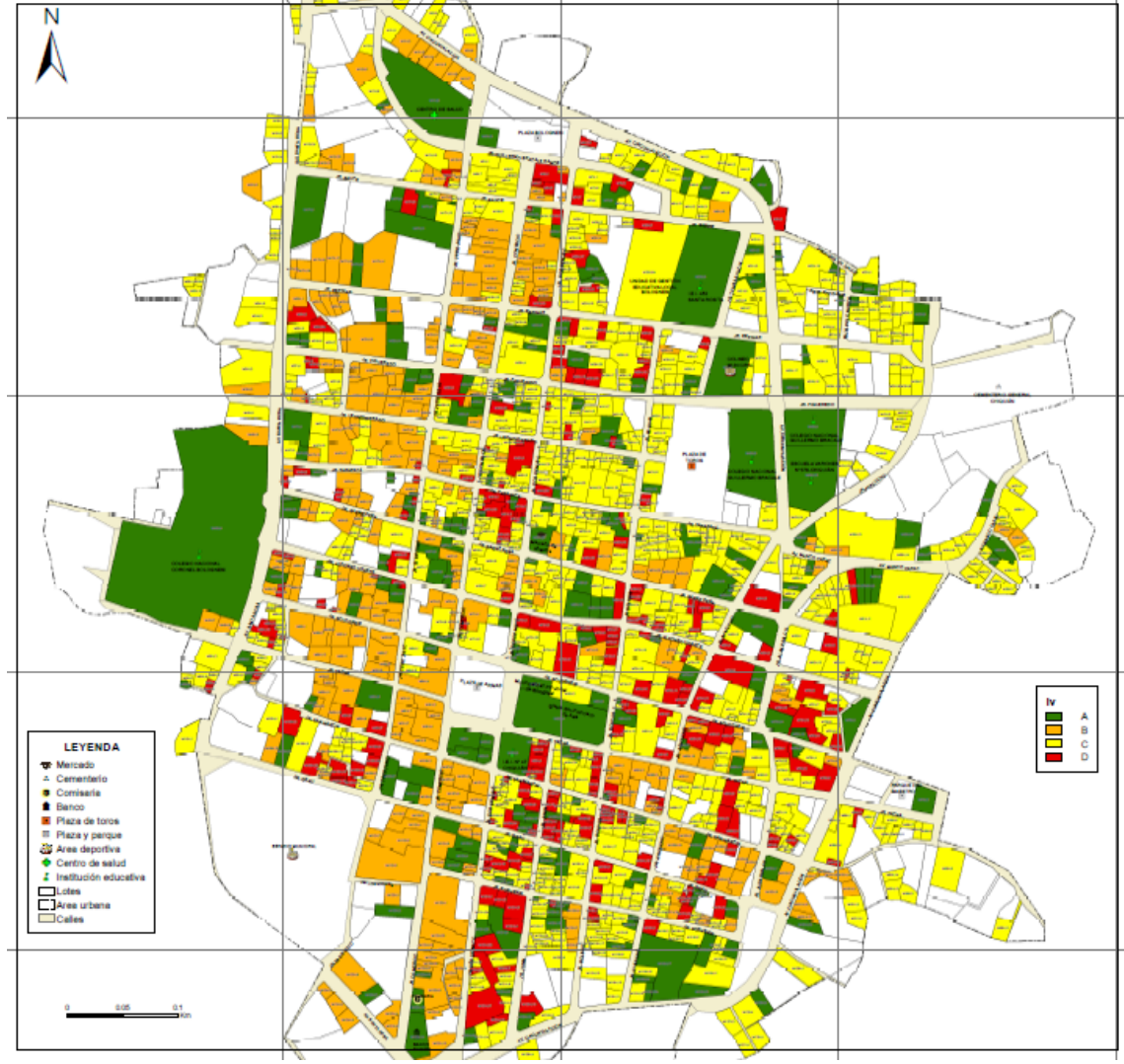

Figura 3. Mapa de índice de vulnerabilidad 


\section{Cálculo del índice de daños}

Es importante destacar que los índices de vulnerabilidad calculados no aportan suficiente información para determinar el riesgo sísmico, por lo que fue necesario transformar estos índices a su equivalente en pérdidas económicas. De acuerdo a (Chavarría y Gómez, 2001), se utilizaron las ecuaciones de las rectas que relacionan el índice de daños para diferentes índices de vulnerabilidad y aceleración horizontal del terreno (a/g). Además, teniendo en cuenta el estudio realizado por (Castillo y Alva, 1993), se consideró para la zona de estudio el valor de la aceleración horizontal a=0,32g. En la siguiente figura se muestra el mapa y el cuadro resumen del índice de daños:

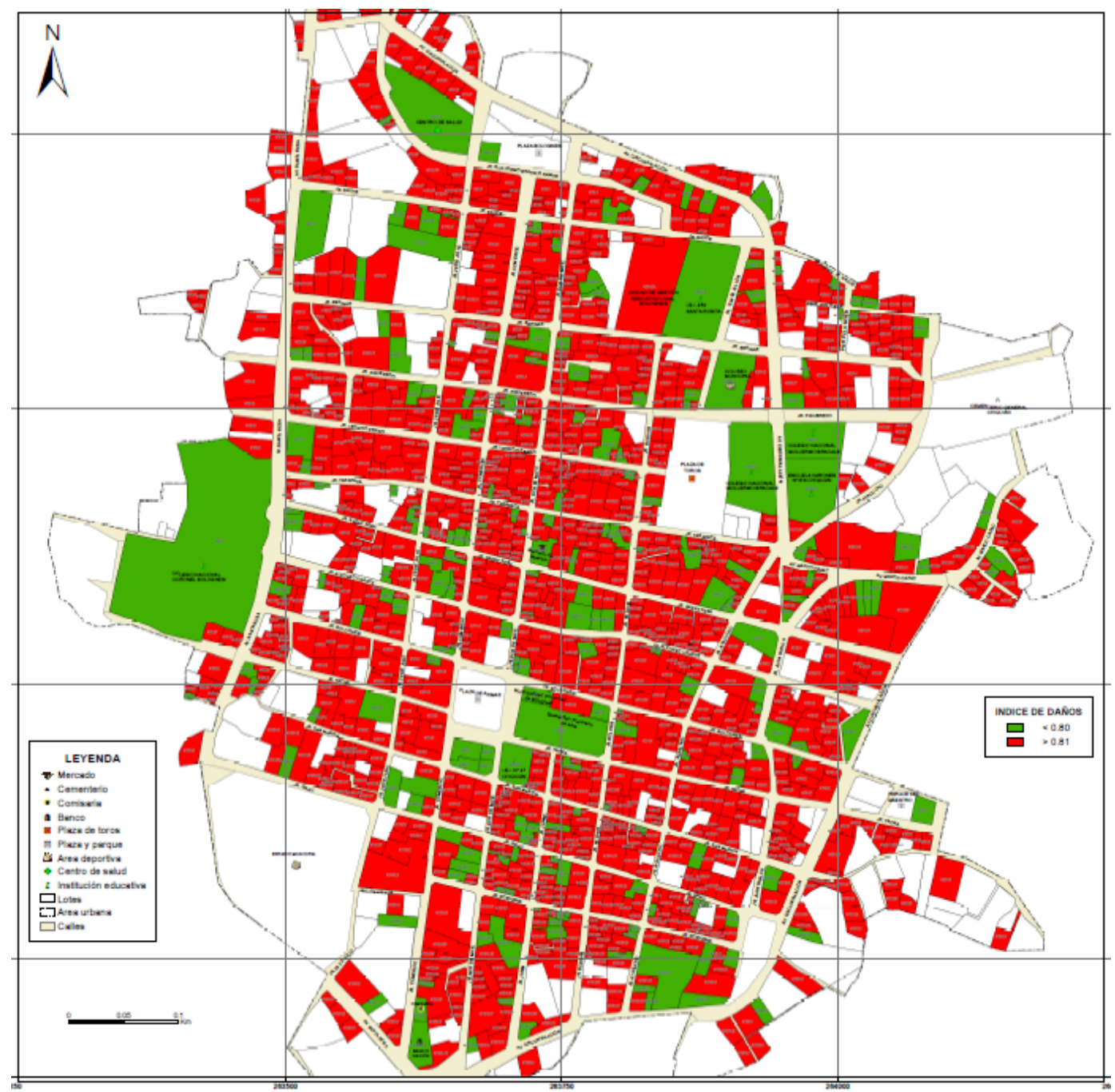

Figura 4. Mapa de índice de daños 
Tabla 3. Resumen de índice de daños

\begin{tabular}{ccc}
\hline Clase & $\mathbf{N}^{\circ}$ viviendas & $\%$ \\
\hline A & 208 & 14.68 \\
B & 1209 & 85.32 \\
Total & $\mathbf{1 4 1 7}$ & $\mathbf{1 0 0 . 0 0}$ \\
\hline
\end{tabular}

Las pérdidas económicas fueron obtenidas teniendo en cuenta que el porcentaje de la edificación que sufre daños es equivalente al índice de daños multiplicado por su área total construida de cada edificación. De acuerdo al informe de la Municipalidad Provincial de Bolognesi el costo aproximado de construcción por metro cuadrado en adobe es de S/ 200,00 y de albañilería es de S/ 500,00. Tomando en cuenta estos valores se ha calculado los daños económicos para las condiciones simuladas de la aceleración horizontal de $0.32 \mathrm{~g}$, encontrándose que en el caso de las edificaciones de adobe los daños económicos cuantificados ascenderían a la suma de S/. 45’935,397.16 y en el caso de las edificaciones de material noble ascenderían a la suma de S/ 24’107,868.98, haciendo un costo total de daños económicos de S/ 70’043,266.14.

\section{DISCUSIÓN}

Teniendo en cuenta que la cantidad de edificaciones evaluadas en la zona urbana del distrito de Chiquián suman 1417, el manejo y análisis de la información geoespacial se torna compleja, por lo que, fue necesario la utilización de los sistemas de información geográfica, los cuales permitieron ordenar, calcular, predecir e interpretar los datos, tal como manifiesta (Flores, 2016). La creación del modelo de vulnerabilidad sísmica, a través de la integración gráfica de diferentes herramientas, permitió la automatización y la sistematización de la información, tal como muestra (Enviromental Systems Research Institute, 2016). La utilización de las nuevas herramientas y tecnologías del SIG en la creación del modelo de vulnerabilidad sísmica, permitirá una adecuada gestión del riesgo en la zona urbana del distrito de Chiquián, a través de su aplicación sencilla, teniendo en cuenta las recomendaciones de (Senouci et al., 2018).

De acuerdo al rango de índice de vulnerabilidad obtenido el 14,70\% de las viviendas se clasifican como vulnerabilidad baja, son viviendas con vigas y columnas de amarre en toda la construcción de la vivienda y son de material noble, con ladrillos procedentes de las fábricas de la ciudad de Lima, cumplen con las normas establecidas en la Norma E.070 del (Ministerio de vivienda, construcción y saneamiento, 2006) y han sido construidas bajo dirección técnica, presentan planos de construcción, distribución regular 
en planta y elevación, se encuentran construidos en terrenos con pendiente baja. En este rango de vulnerabilidad se encuentran la mayoría de las nuevas construcciones de material noble, con una antigüedad de 0 a 5 años y las infraestructuras de servicio público, tal como muestra (Saputra et al., 2017) en su investigación.

El 21,20\% de las viviendas se encuentran clasificadas en vulnerabilidad Media, cumplen con algunas características del Reglamento Nacional de Edificaciones, Ministerio de Vivienda, Construcción y Saneamiento (2006), son viviendas de material noble y en su mayoría de adobe con vigas y columnas de amarre con ciertas deficiencias, son de material noble y han sido construidas sin dirección técnica, con ladrillos artesanales que no cumplen con la resistencia técnica establecida, presentan algunas irregularidades en planta y elevación, presentan diafragmas horizontales y son viviendas con un antigüedad mayor a 10 años.

El 48,80\% de las viviendas presentan una vulnerabilidad Alta, es decir presentan algunas características mencionadas en el Reglamento Nacional de Edificaciones, son viviendas de barro que no presentan vigas y columnas de amarre que se encuentran distribuidos a lo largo de la zona urbana, son de adobe fabricados en forma artesanal, no son de buena calidad y su construcción se ha realizado sin la presencia de mano de obra calificada, presentan irregularidades en planta y elevación y no poseen planos de construcción, según se muestra en las figuras 5 y 6 .

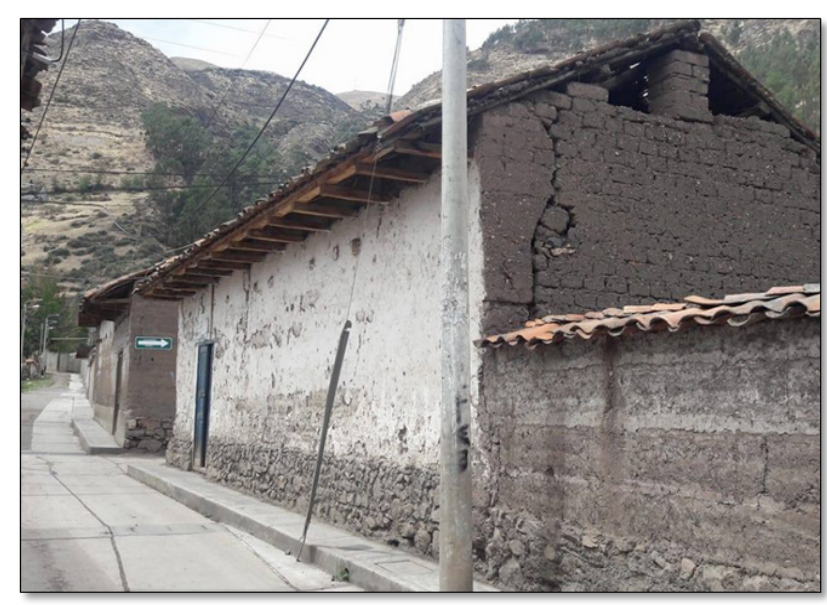

Figura 5. Vivienda de adobe con rajaduras de muros

El 15,20\% de las viviendas presentan una vulnerabilidad Muy Alta, debido a que no cumplen con el Reglamento Nacional de Edificaciones, Ministerio de Vivienda, Construcción y Saneamiento (2006), es decir no presentan vigas y columnas de amarre, son viviendas de adobe con una antigüedad mayor a 30 años, que se encuentran en desuso, 
pero continúan siendo habitadas. Por otro lado, presentan irregularidades en planta y elevación, no presentan planos de construcción y fueron ejecutados por personas empíricas, sin dirección técnica, similar a los resultados obtenidos por (Saputra et al., 2017).

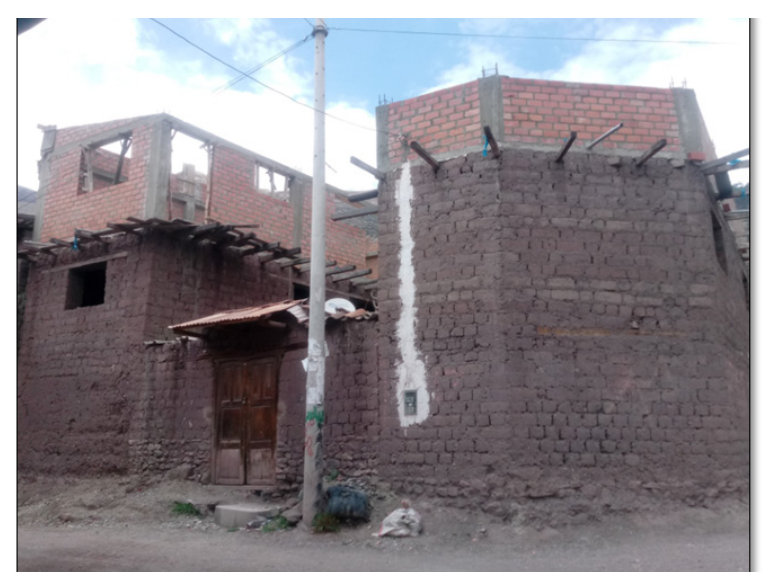

Figura 6. Vivienda de adobe soportando al tercer nivel de ladrillo

\section{CONCLUSIONES}

El modelo de vulnerabilidad sísmica realizado con la herramienta model builder del ArcGIS permitió la automatización y obtención del mapa de índice de vulnerabilidad sísmica para la zona urbana de Chiquián, pudiendo ser replicado en cualquier estudio de esta naturaleza.

El análisis de los once parámetros y el cálculo del índice de vulnerabilidad muestran que el 14,7\% de las viviendas se encuentran en vulnerabilidad baja (clase A), el 21,2\% se encuentran en vulnerabilidad media (clase B), el 48,8\% se encuentran en vulnerabilidad alta (clase C) y el 15,2\% se encuentran en vulnerabilidad muy alta (clase D).

Teniendo en cuenta el índice de vulnerabilidad y una aceleración horizontal del terreno de $0,32 \mathrm{~g}$, se observa que 1209 viviendas $(85,32 \%)$ sufrían el colapso total y 208 viviendas $(14,68 \%)$ seguirían en pie con algún daño estructural.

De acuerdo al índice de daño calculado y teniendo en cuenta el precio por metro cuadrado de construcción, si ocurriera un sismo con las características descritas anteriormente los daños económicos ascenderían a la suma de S/ 70’043,266.14.

\section{AGRADECIMIENTO}

A la Municipalidad Provincial de Bolognesi, en nombre de su alcalde Ing. Aníbal Bazán Alvarado. A los Bachilleres en Ingeniería Agrícola José Carrera, Clinton Guerrero y Nelson Villacorta por su apoyo en el levantamiento de información. 


\section{REFERENCIAS BIBLIOGRÁFICAS}

Castillo, Jorge y Jorge, Alva. 1993. «Peligro sísmico en el Perú.» Último acceso: < http:/ / www.jorgealvahurtado.com/files/redacis15_a.pdf>. [Consulta: 10-10-2017].

Chavarría, Daniel y Daniel, Gómez . 2001. Estudio de vulnerabilidad sísmica en viviendas de 1 y 2 pisos del barrio Cuarto de Legua en el cono Cañaveralejo. Tesis de pregrado. Universidad del Valle, Santiago de Cali.

De la Cruz, Italo. 2011. Peligro sísmico probabilístico y espectro uniforme en la Región de Ancash. Tesis. Huaraz, Ancash. <https://es.scribd.com/doc/85939015/ Peligro-Sismico-Ancash-PERU-Tesis-2012-PSHA-UHS\#>. [Consulta: 09-112017].

Enviromental Systems Research Institute. 2016. ArcGIS for Desktop. <http://desktop.arcgis.com/es/arcmap/10.3/analyze/modelbuilder/what-is-modelbuilder. htm>. [Consulta: 14-11-2017].

Flores, Ricardo. 2016. Modelamiento geoespacial para la determinación del grado de vulnerabilidad, distrito Leimebamba-Amazonas. Tesis. Universidad Nacional Agraria La Molina, Lima.

Marín, Félix. 2012. Evaluación del riesgo sísmico del centro histórico de la Ciudad de Huánuco. Tesis de grado. Huánuco. Último acceso: < http://cybertesis.uni.edu. pe/handle/uni/1236>. [Consulta: 22-11-2018].

Ministerio de Vivienda, Construcción y Saneamiento. 2016. «Decreto supremo que modifica la Norma Técnica E.030 "Diseño sismorresistente" del Reglamento Nacional de Edificaciones.» Lima. <file:// C:/Users/USER/Downloads/DS003-2016-VIVIENDA.pdf> . [Consulta: 22-11-2018].

Ministerio de vivienda, construcción y saneamiento. 2006. «Norma E.070: Albañilería.» <http://blog.pucp.edu.pe/blog/wp-content/uploads/sites/82/2008/01/ Norma-E-070-MV-2006.pdf>. [Consulta: 22-11-2018].

Saputra, Aditya et al. 2017. «Seismic vulnerability assessment of residential buildings using logistic regression and geographic information system (GIS) in Pleret Sub District (Yogyakarta, Indonesia)». Geoenvironmental Disasters. <https://link.springer.com/article/10.1186/s40677-017-0075-z\#Sec6> [Consulta: 22-11-2018].

Senouci, Abbas et al. 2018. «Mapping seismic vulnerability at urban scale: Discussion on relevant cartography representations and smoothing for urban planning purposes on the Oran case study.» Soil Dynamics and Earthquake Engineering 545-563. <https://www.sciencedirect.com/science/article/pii/S026772611730667X>. [Consulta: 22-11-2018].

Recepción: 05/06/2018

Aceptación: 30/11/2018

\section{Correspondencia}

Tito Tinoco Meyhuay

ttinoco2409@hotmail.com 